\title{
Opposing effects of nitric oxide and prostaglandin inhibition on muscle mitochondrial $\dot{\mathrm{V}}_{\mathrm{O}_{2}}$ during exercise
}

\author{
Robert Boushel, ${ }^{1,4}$ Teresa Fuentes, ${ }^{2}$ Ylva Hellsten, ${ }^{3,4}$ and Bengt Saltin ${ }^{4}$ \\ ${ }^{1}$ Heart and Circulatory Unit, Department of Biomedical Sciences; Mitochondrial Research Laboratory, Department of \\ Anaesthesia Bispebjerg Hospital, University of Copenhagen, Copenhagen, Denmark; ${ }^{2}$ University of Las Palmas de Gran \\ Canaria, Las Palmas, Spain; ${ }^{3}$ Institute of Exercise and Sport Sciences, University of Copenhagen, Copenhagen, Denmark; \\ and ${ }^{4}$ The Copenhagen Muscle Research Centre, Copenhagen, Denmark
}

Submitted 31 January 2012; accepted in final form 18 April 2012

Boushel R, Fuentes T, Hellsten Y, Saltin B. Opposing effects of nitric oxide and prostaglandin inhibition on muscle mitochondrial $\dot{\mathrm{V}}_{2}$ during exercise. Am J Physiol Regul Integr Comp Physiol 303: R94-R100, 2012. First published May 2, 2012; doi:10.1152/ajpregu.00044.2012.-Nitric oxide (NO) and prostaglandins (PG) together play a role in regulating blood flow during exercise. NO also regulates mitochondrial oxygen consumption through competitive binding to cytochrome- $c$ oxidase. Indomethacin uncouples and inhibits the electron transport chain in a concentration-dependent manner, and thus, inhibition of NO and PG synthesis may regulate both muscle oxygen delivery and utilization. The purpose of this study was to examine the independent and combined effects of NO and PG synthesis blockade (L-NMMA and indomethacin, respectively) on mitochondrial respiration in human muscle following knee extension exercise (KEE). Specifically, this study examined the physiological effect of $\mathrm{NO}$, and the pharmacological effect of indomethacin, on muscle mitochondrial function. Consistent with their mechanism of action, we hypothesized that inhibition of nitric oxide synthase (NOS) and PG synthesis would have opposite effects on muscle mitochondrial respiration. Mitochondrial respiration was measured ex vivo by high-resolution respirometry in saponin-permeabilized fibers following 6 min KEE in control $(\mathrm{CON} ; n=8)$, arterial infusion of $N^{\mathrm{G}}$-monomethyl-L-arginine (L-NMMA; $\left.n=4\right)$ and Indo $(n=4)$ followed by combined inhibition of NOS and PG synthesis (L-NMMA + Indo, $n=8$ ). ADP-stimulated state 3 respiration (OXPHOS) with substrates for complex I (glutamate, malate) was reduced $50 \%$ by Indo. State $3 \mathrm{O}_{2}$ flux with complex I and II substrates was reduced less with both Indo (20\%) and L-NMMA + Indo (15\%) compared with $\mathrm{CON}$. The results indicate that indomethacin reduces state 3 mitochondrial respiration primarily at complex I of the respiratory chain, while blockade of NOS by L-NMMA counteracts the inhibition by Indo. This effect on muscle mitochondria, in concert with a reduction of blood flow accounts for in vivo changes in muscle $\mathrm{O}_{2}$ consumption during combined blockade of NOS and PG synthesis.

$N^{\mathrm{G}}$-monomethyl-L-arginine; indomethacin; contraction; OXPHOS; oxygen uptake

NITRIC OXIDE (NO) AND PROSTAGLANDINS (PG) contribute synergistically to regulate skeletal muscle blood flow during exercise in humans $(5,14,15,28,32,33)$ through endothelium-dependent relaxation of vascular smooth muscle. Some studies employing dual inhibition of NO and PG synthesis have reported an effect on muscle oxidative metabolism and energy cost of contraction $(14,15,28)$, suggesting overlapping regulation of oxygen delivery and utilization.

NO is an endogenous, short-lived free radical that mediates vascular smooth muscle relaxation. At low physiological con-

Address for reprint requests and other correspondence: R. Boushel, Dept. of Anaesthesia, Bispebjerg Hospital, Bldg. 60, 23 Bispebjerg Bakke, 2400 Copenhagen NV, Denmark (e-mail: boushel@sund.ku.dk). centrations (nanomolar range), NO also reversibly inhibits mitochondrial oxygen consumption by competitive binding to the heme $\mathrm{a}_{3}$ site of cytochrome oxidase in competition with oxygen. Higher ( $\mathrm{mM}$ ) concentrations of $\mathrm{NO}$ leads to peroxinitrite production that, in turn, irreversibly inhibits complexes I and II of the electron transport chain $(6-9,34,37,43)$. Indomethacin is a nonsteroidal anti-inflammatory drug (NSAID) that inhibits cyclooxygenase-mediated formation of PG from arachidonic acid and PG-mediated vasodilation. NSAIDs are hydrophobic acids that release protons from amino, carboxyl, and hydroxyl groups, which, therefore, can also act as ionophores in the inner mitochondrial membrane, resulting in uncoupling of substrate oxidation from ATP production (21, 25-27). At high concentrations, NSAIDs interact with mitochondrial membrane phospholipids, resulting in inhibition of mitochondrial electron transport and ATP production and have been shown to exert a negative inotropic effect on cardiac contractility (21, 26, 27).

Studies examining mechanisms of blood flow control have revealed conflicting findings on whether $\mathrm{NO}$ increases or decreases $\dot{\mathrm{V}}_{2}$ during contraction. In animals, NOS inhibition has been shown to lower muscle $\dot{\mathrm{V}}_{2}$ during evoked contractions $(2,20,22,30,31,41)$, while other studies show no effect $(1,4)$ or an increase $(35)$ in muscle $\dot{\mathrm{V}}_{2}$ during voluntary exercise. In humans, NOS inhibition alone has been found to have little or no effect on $\dot{\mathrm{V}}_{2}$ across the exercising leg or forearm measured by the Fick method $(15,28,33)$. This contrasts a recent study demonstrating that dietary nitrate that increases the bioavailability of NO reduces oxygen cost during physical exercise accompanied by improvement in mitochondrial oxidative phosphorylation efficiency ( $\mathrm{P} / \mathrm{O}$ ratio) and a decrease in state 4 respiration measured ex vivo in isolated mitochondria (23). Another study showed that muscle $\dot{\mathrm{V}}_{2}$ measured by $\mathrm{PET}$ with radio-water and radioinhaled oxygen was increased at rest with NOS inhibition and higher during exercise with combined inhibition of NOS and PG synthesis (14).

To gain further insight into the mechanisms underlying how muscle $\dot{\mathrm{V}}_{2}$ is affected during exercise by NOS and PG synthesis inhibition in humans, mitochondrial respiration was measured in permeabilized fibers from muscle biopsies taken immediately after knee extension exercise with and without arterial infusion of $N^{\mathrm{G}}$-monomethyl-L-arginine (L-NMMA) and/or indomethacin. Accordingly, the study examined two separate regulatory effects on mitochondria, the "physiological" inhibitory effect of NO, and the pharmacological effect of indomethacin on the electron transport chain. 
NOS inhibition was expected to increase muscle mitochondrial respiration by reducing the NO inhibition of cytochrome- $c$ oxidase, while NSAID treatment was expected to decrease mitochondrial respiration because of the intrinsic NSAID effect on complex I, rather than due to PG synthesis inhibition. Consistent with their mechanism of action, we hypothesized that inhibition of NOS and PG synthesis would have opposite effects on muscle mitochondrial respiration.

\section{METHODS}

Human subjects. Eight healthy males, aged $24 \pm 2 \mathrm{yr}$, height $175 \pm$ $3 \mathrm{~cm}$, and weight $71 \pm 2 \mathrm{~kg}$, volunteered to participate in the study. All subjects were physically active but not involved in organized training for any sport, and reported no recent history of regular exercise training. Subjects were informed about the possible risks and discomforts involved in the study before giving their written consent to participate. This study was carried out according to the Declaration of Helsinki and was approved by the Ethical Committee of Copenhagen (KF 11-042/04).

On the day of the experiment, subjects arrived at the laboratory 1 $\mathrm{h}$ prior to the experiment after a light breakfast. A catheter was placed into the femoral artery of the right leg under local anesthesia. Following $30 \mathrm{~min}$ of rest, the subject performed one-leg knee extension exercise at $30 \mathrm{~W}$ under the following three conditions: 1) Control $(\mathrm{CON}), 2)$ either L-NMMA infusion or indomethacin (Indo) infusion, followed by 3) L-NMMA + Indo (double blockade) infusion. Each exercise bout was separated by a 45 -min rest period. All eight subjects performed CON and L-NMMA + Indo, while subjects were randomized to perform an additional bout with either L-NMMA ( $n=$ 4) or Indo $(n=4)$. Saline, Indo $\left(120 \mu \mathrm{g} \cdot \mathrm{min}^{-1} \cdot \mathrm{kg}^{-1}\right.$ of leg mass; Alphapharma), and/or L-NMMA (4 $\mathrm{mg} \cdot \mathrm{min}^{-1} \cdot \mathrm{kg}^{-1}$ of leg mass; Clinalfa, Bachem) were infused into the femoral artery for $4 \mathrm{~min}$ before exercise and were then infused at a rate of $60 \mu \mathrm{g} \cdot \mathrm{min}^{-1} \cdot \mathrm{kg}^{-1}$ of leg mass (Indo) and/or $2 \mathrm{mg} \cdot \mathrm{min}^{-1} \cdot \mathrm{kg}^{-1}$ of leg mass (L-NMMA; maintenance dose) during the 6 min of active exercise. The dosages of L-NMMA and indomethacin infused were selected on the basis of previous experiments that found a reduction in muscle blood flow during exercise (28).

Skeletal muscle tissue. After local anesthesia (1\% Lidocaine) of the skin and superficial muscle fascia, biopsies were obtained from the vastus lateralis muscle immediately after exercise in all four conditions; CON; L-NMMA; Indo; L-NMMA + Indo. A small portion of the biopsied muscle $(\sim 2-3 \mathrm{mg})$ was placed in relaxing medium (10 mM Ca-EGTA buffer, $0.1 \mu \mathrm{M}$ free calcium, $20 \mathrm{mM}$ imidazole, 20 $\mathrm{mM}$ taurine, $50 \mathrm{mM}$ K-MES, $0.5 \mathrm{mM}$ DTT, $6.56 \mathrm{mM} \mathrm{MgCl}_{2}, 5.77$ $\mathrm{mM}$ ATP, and $15 \mathrm{mM}$ phosphocreatine, $\mathrm{pH} 7.1$ ) at $2-4^{\circ} \mathrm{C}$. Fiber bundles were gently separated under a dissecting microscope with a pair of pointed forceps to maximize surface area of each fiber. The fiber bundles were then permeabilized for $30 \mathrm{~min}$ in $3 \mathrm{ml}$ of ice-cold relaxing medium with saponin $(50 \mu \mathrm{g} / \mathrm{ml})$. After chemical permeabilization, the tissue was rinsed in chilled solution of $\mathrm{MgCl}_{2} \cdot 6 \mathrm{H}_{2} \mathrm{O}$ (3 $\mathrm{mM})$, K-lactobionate $(60 \mathrm{mM})$, taurine $(20 \mathrm{mM}), \mathrm{KH}_{2} \mathrm{PO}_{4}(10 \mathrm{mM})$, HEPES $(20 \mathrm{mM})$, sucrose $(110 \mathrm{mM})$, BSA $(1 \mathrm{~g} / \mathrm{l})$, at $\mathrm{pH}$ 7.1. The muscle bundles were then blotted and measured for wet weight in a balance. The muscle bundles were then transferred immediately into a respirometer (Oxygraph-2k, Oroboros Instruments, Innsbruck, Austria) containing the medium respiration (as above) at a chamber temperature of $37^{\circ} \mathrm{C}$.

Respirometry. The Oxygraph-2k is a two-chamber titration-injection respirometer with a limit of oxygen flux detection of 1 $\mathrm{pmol} \cdot \mathrm{s}^{-1} \cdot \mathrm{ml}^{-1}$. Standardized instrumental and chemical calibrations were performed to correct for back diffusion of oxygen into the chamber, leak from the exterior, and oxygen consumption by the chemical medium and $\mathrm{O}_{2}$ sensor. $\mathrm{O}_{2}$ flux of muscle fibers was resolved by software (Datlab 4, Oroboros Instruments, Innsbruck,
Austria), allowing nonlinear changes in the negative time derivative of the oxygen concentration signal. Resting respiration (state 2; absence of adenylates) for complex I of the mitochondrial respiratory chain was assessed by addition of malate $(2 \mathrm{mmol} / \mathrm{l})$ and glutamate (10 $\mathrm{mmol} / \mathrm{l})$, followed by ADP-stimulated $(5 \mathrm{mmol} / \mathrm{l})$ coupled state 3 respiration. The addition of succinate $(10 \mathrm{mmol} / \mathrm{l})$ elicited state 3 respiration with convergent electron input to both complexes I and II. Uncoupled respiratory rate was assessed by titration of FCCP $(0.7$ $\mu \mathrm{mol} / \mathrm{l})$. In an additional experiment using a separate portion of the CON muscle, state 3 respiration with complex I and II substrates (2 $\mathrm{mmol} / \mathrm{l}$ malate $+10 \mathrm{mmol} / \mathrm{l}$ glutamate $+10 \mathrm{mmol} / \mathrm{l}$ succinate +5 $\mathrm{mmol} / \mathrm{l} \mathrm{ADP}$ ) was induced followed by dose-response experiments with sequential titration of indomethacin $(0.1-1.3 \mathrm{mM})$ and L-arginine $(0-500 \mu \mathrm{M})$ into the respirometry chamber.

Animal experiments. To explore physiological gain and loss of function of $\mathrm{NO}$ on mitochondrial respiration, experiments on rat soleus muscle $(n=8)$ were undertaken to measure the effects of the NOS substrate L-arginine (Sigma-Aldrich, St. Louis, MO) with and without L-NMMA on mitochondrial state 3 respiration. This experiment also tested the intactness of the enzymatic production of NO by NOS and whether or not the ex vivo permeabilization and rinsing procedures had a washout effect on the enzyme. The animals were killed under proper euthanasia, and a portion of the soleus muscle was surgically removed immediately from the rat hindlimb and placed in relaxing medium. Muscles were dissected and permeabilized as described above. State 3 respiration with malate $(2 \mathrm{mmol} / \mathrm{l})+$ glutamate $(10$ $\mathrm{mmol} / \mathrm{l})+$ succinate $(10 \mathrm{mmol} / \mathrm{l})+\operatorname{ADP}(5 \mathrm{mmol} / \mathrm{l})$ was measured followed by successive titrations of L-arginine (25-500 $\mu \mathrm{mol} / \mathrm{l})$, with and without L-NMMA (1 mM). Experiments were approved by the Danish Animal Experimental Inspectorate and complied with the European convention for the protection of vertebrate animals used for experiments and other scientific purposes (Council of Europe, no. 123, Strasbourg, France, 1985).

Data analysis. Comparison of different respiratory states was analyzed using a one-way ANOVA with repeated measures for isolated or combined drug interventions (Sigma Stat 3.0). Data are presented as means \pm SE. Significance was accepted at $P<0.05$.

\section{RESULTS}

Human experiments. ADP-stimulated state 3 mitochondrial respiration (also known as OXPHOS) with substrates for complex I (glutamate and malate) was reduced by Indo (Fig. 1A) compared with all other conditions $(\mathrm{CON}, 52 \%$; Double Block $63 \%$, and L-NMMA $86 \%, P<0.05)$. No differences were observed between CON and Double Block $(P=0.674)$, while L-NMMA tended to be higher than $\operatorname{CON}(P=0.10)$. State $3 \mathrm{O}_{2}$ flux with convergent electron input through both complex I and II, with addition of succinate was similarly reduced with Indo $(20 \%)$ and Double Block $(15 \%)(P<0.001)$ compared with CON but was not lowered with L-NMMA (Fig. 1B). The substrate control ratio (SCR) indicating the ratio between complex I + II: complex I (GMS3/GM3) was significantly higher in Indo muscle compared with all other conditions, demonstrating the disproportionately large inhibition of complex I by Indo (Fig. 2A). The lower SCR in Double Block and L-NMMA $(P<0.05)$ confirms a permissive effect of NOS inhibition on complex I respiration.

Uncoupled $\mathrm{O}_{2}$ flux determined by titration of the ionophore FCCP was not increased above state 3 respiration with Indo, indicating inhibition of full electron transport chain capacity by Indo (Fig. 2B). Uncoupled respiration (3U/GS3) was higher in Double Block $(P<0.05)$ and L-NMMA $(P<0.01)$, indicating tight phosphorylation control in state 3 and a higher electron transport capacity with inhibition of NOS, consistent with an 
A

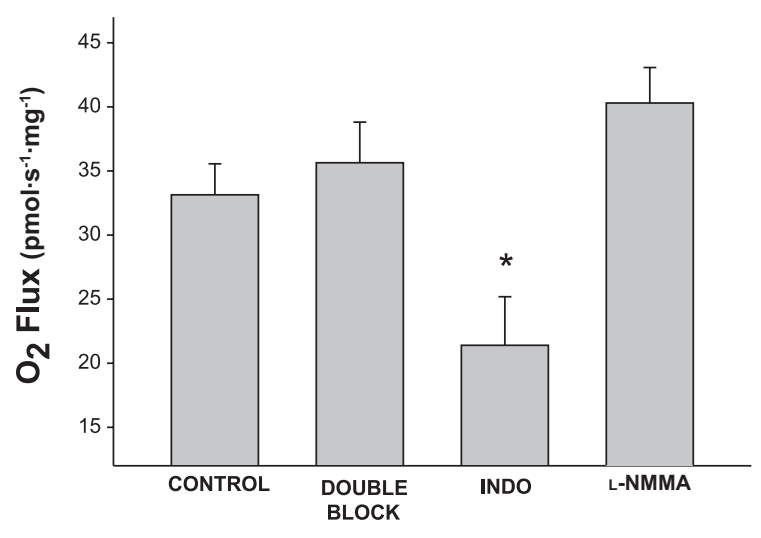

B

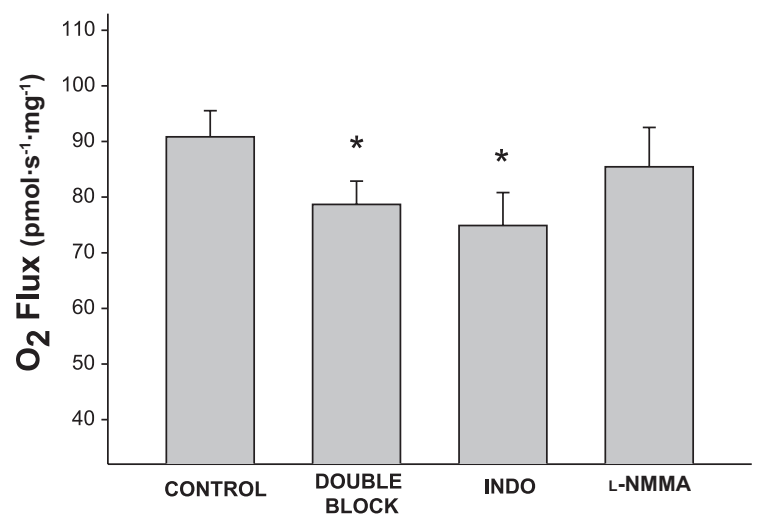

Fig. 1. A: state $3 \mathrm{O}_{2}$ flux with electron input to complex I (malate, glutamate, ADP) in CON, Indo, L-NMMA, and double block. B: state 3 respiration for complex I + II (malate, glutamate, succinate, and ADP). *Significant difference, $P<0.05$ from control.

effect on cytochrome- $c$ oxidase. This response was also higher than Indo $(P<0.05$ and $P<0.01)$. There was no limitation on mitochondrial state 3 respiration by ATP synthetase after exercise in Indo and CON, with a UCR of 0.99 and 1.04, respectively, and there was a small but significant limitation of the electron transport chain by ATP synthetase with L-NMMA $(\mathrm{UCR}=1.1)$ and Double Block $(\mathrm{UCR}=1.13)$. These findings also indicate an inhibitory effect of $\mathrm{NO}$ on the electron transport chain in human skeletal muscle. In separate dose-response experiments with a portion of the human muscle biopsy, graded titration of indomethacin into the respirometry chamber containing CON muscle caused a linear reduction in state 3 respiration $(P<0.001)$ within and above the physiological range (Fig. 3).

Animal experiments. The effect of adding L-arginine, the substrate for NOS with and without NOS inhibition on state 3 respiration in rat skeletal is shown in Fig. 4. State 3 respiration with complex I + II electron supply was reduced with L-arginine compared with CON, while NOS inhibition (L-NMMA + L-arginine) increased state 3 and $\mathrm{COX} \mathrm{O}_{2}$ flux (Fig. 5).

\section{DISCUSSION}

In this study, we measured regulatory aspects of mitochondrial respiration in permeabilized fibers from biopsies of the vastus lateralis following exercise to gain insights into the mechanisms underlying previously reported changes in local muscle blood flow and $\mathrm{V}_{2}$ in vivo with vasodilator inhibition. The main findings of this study are that 1 ) arterial infusion of indomethacin suppresses state 3 mitochondrial respiration primarily at complex I of the respiratory chain and 2) inhibition of nitric oxide synthase by L-NMMA increases mitochondrial electron transport capacity and counteracts the limitation of state 3 respiration by indomethacin. These findings confirm the hypothesis that inhibition of NOS increases mitochondrial state 3 respiration, while indomethacin exerts a pharmacological effect of inhibition of mitochondrial respiration. In the muscle samples taken after arterial infusion of indomethacin, state 3 mitochondrial respiration was reduced by $50 \%$ with mitochondrial complex I substrates (Fig. 1A). This finding is consistent with other reports of complex I-specific inhibition by indomethacin $(16,25-27,36)$. Although it is known that low-dose indomethacin exerts an uncoupling effect in various cell types $(21,24,25,27)$, the results of this study from either the infusion experiment in vivo (Figs. 1 and 2) or the additional
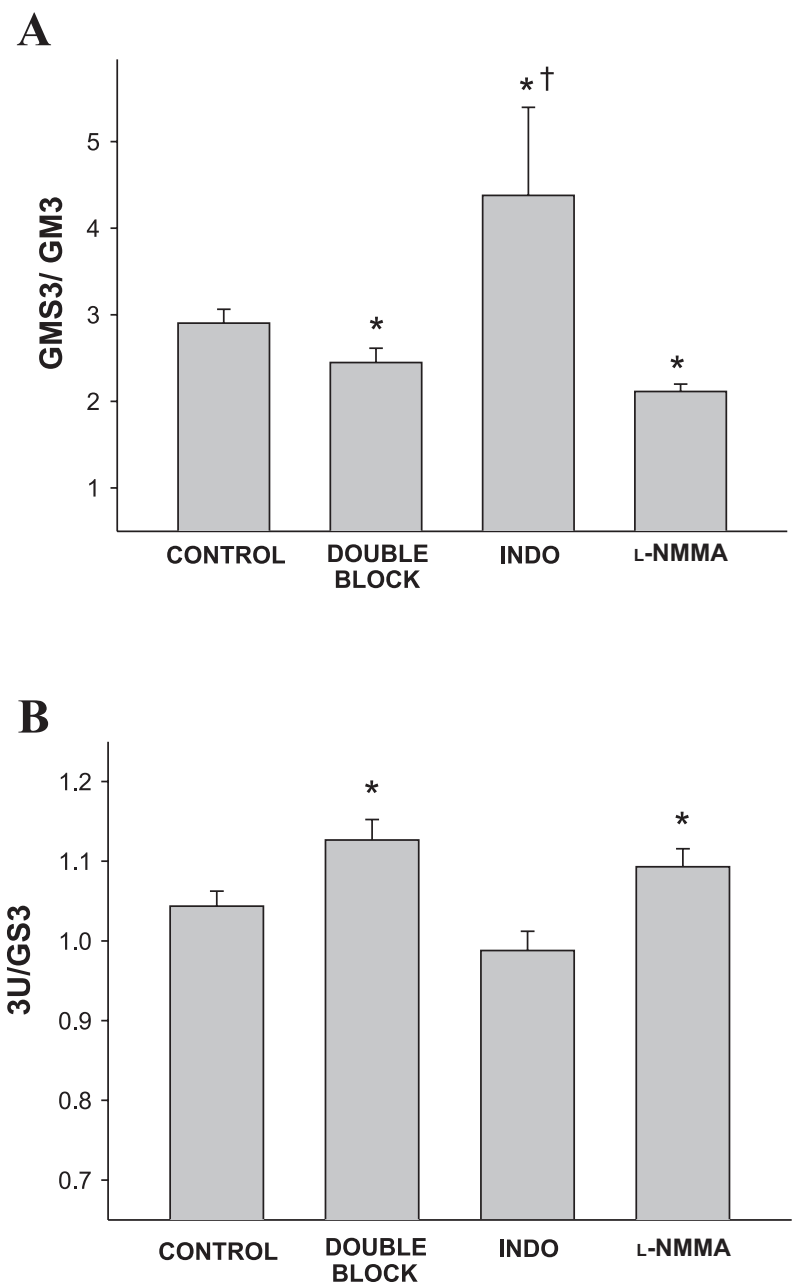

Fig. 2. A: ratio between complex I + II: complex I (GMS3/GM3) between state $3 \mathrm{O}_{2}$ flux with convergent electron input through both complex I (glutamate and malate) and II with addition of succinate vs. $\mathrm{O}_{2}$ flux through only complex I in control, $N^{\mathrm{G}}$-monomethyl-L-arginine (L-NMMA) + Indo (double blockade), Indo and L-NMMA. $B$ : uncoupling control ratio (UCR) reflecting the ratio increase of uncoupled respiration above coupled state respiration in control, double block, Indo, and L-NMMA. *Significant difference control, $P<0.05$. $\dagger$ Significant difference for Indo vs. double block, $P<0.05$. 


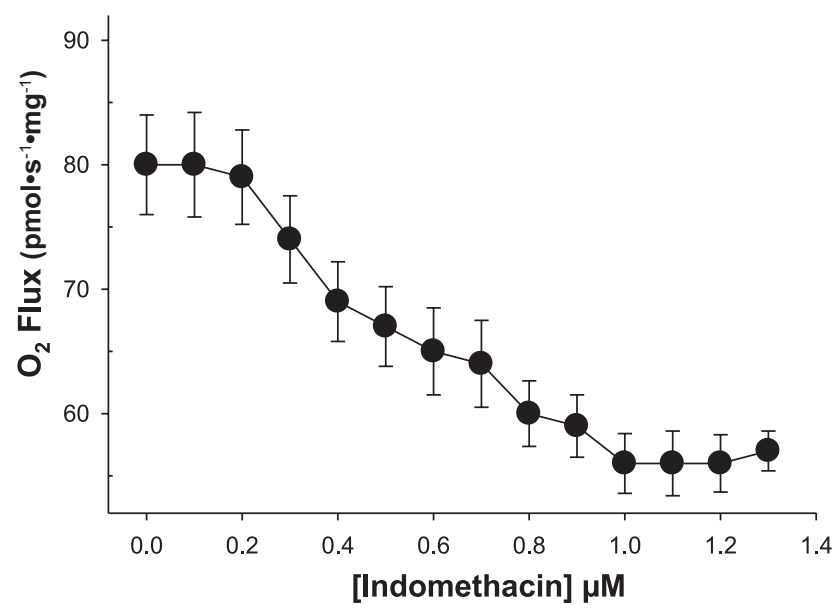

Fig. 3. Dose-response effect of indomethacin and on state 3 mitochondrial respiration with electron supply to complex I + II in control muscle fibers in humans.

dose-response experiment (Fig. 3) do not support an uncoupling effect in skeletal muscle that would increase mitochondrial $\dot{\mathrm{V}}_{2}$. In contrast, the present findings are consistent with reports showing that indomethacin disrupts complex I of electron transport chain by mechanisms linked to leakage of electrons, formation of superoxide anions $\left(\mathrm{O}_{2}{ }^{-}\right)$, iron release from aconitase, and hydroxyl radical formation $(12,13,20,40$, 43). When NOS blockade with L-NMMA was then combined with indomethacin, there was no difference in complex I respiration compared with $\mathrm{CON}$, indicating that during the indomethacin condition alone, NO bioavailability exerts permissive inhibition of complex I to suppress mitochondrial respiration by downstream control of cytochrome- $c$ oxidase. State $3 \mathrm{O}_{2}$ flux with addition of the complex II substrate succinate to provide convergent electron input to the Q-junction was less affected with Indo (20\%) and similar to Double Block $(15 \%)(P<0.001)$ compared with $\mathrm{CON}$ and tended to increase with L-NMMA alone (Fig. $1 B$ ). This recovery of respiration with L-NMMA is likely due to the lower NO concentration and lower $K_{\mathrm{m}}$ of cytochrome- $c$ oxidase for $\mathrm{O}_{2}$, allowing greater electron flux through complex II, which has a lower P:O ratio and thus a higher oxygen consumption for a given molar equivalent of substrate. This is supported by the significantly higher substrate control ratio (GMS3/GM3, Fig. $2 A$ ) with Indo, indicating a proportionately greater contribution of complex II substrate to total respiration.

Maximal electron transport capacity was evaluated with the inner mitochondrial membrane uncoupler FCCP in the presence of complex I and II substrates. The inhibitory effect of Indo on the respiratory chain is apparent from Fig. $2 B$, where the ratio of FCCP to state 3 respiration (uncoupling control ratio, UCR) was lower than 1.0, in contrast to the other conditions, illustrating control of the respiratory chain by the phosphorylation system. This pattern reflects tight coupling of respiration to ATP synthase in CON and shows a lower electron transport through the respiratory chain with indomethacin. This finding together with the lower state 3 respiration rate (Fig. 1A) confirms the inhibition of electron transport by indomethacin. The finding that NOS blockade (both Double Block and L-NMMA) resulted in a higher UCR compared with CON (Fig. 2B), provides further evidence of an interaction between NO and Indo in the regulation of mitochondrial respiration, i.e., limiting electron transport chain capacity. Thus, NOS inhibition increases state 3 mitochondrial respiration and alleviates the inhibitory effect of indomethacin and NO on the electron transport system. The results from the animal experiments in this study confirm our finding in humans that NO exerts an inhibitory effect on cytochrome- $c$ oxidase and on state 3 respiration. There was a significant doseresponse inhibition of state 3 respiration with titration of incremental doses of L-arginine (Fig. 4). Inhibition of NOS with L-NMMA alleviated this suppression, such that state $3 \mathrm{O}_{2}$ flux was higher than both the NO donor (L-arginine) and CON experiments (Fig. 5). The animal experiments also confirm the results from the in vivo infusion experiments that $\mathrm{NO}$ availability for cytochrome- $c$ oxidase can exert feedback flux control of OXPHOS. This is consistent with other reports on cytochrome- $c$ oxidase regulation of upstream electron flux in human muscle where mitochondrial $\mathrm{O}_{2}$ flux rates at rest and during exercise correlate strongly to the mitochondrial oxygen affinity ( $p_{50}$ mito) $(23)$.

Studies in isolated hindlimb and diaphragm muscle in dogs and rats have reported a lower muscle $\dot{\mathrm{V}}_{2}$ during highintensity evoked contractions with $\operatorname{NOS}$ inhibition $(2,20,22$, $31,41,42)$. These findings have been interpreted as an $\mathrm{O}_{2}$ delivery limitation owing to reduced muscle blood flow. Consistent with this interpretation, there are reports of no effect (1, 4) or an increase (35) in muscle $\mathrm{V}_{2}$ during "submaximal" exercise in animals where increased $\mathrm{O}_{2}$ extraction compensates for a lower blood flow. In humans, NOS inhibition alone has no effect on $\dot{\mathrm{V}}_{2}$ across the exercising leg, but when combined with PG synthesis, inhibition by indomethacin, a significant reduction in blood flow is compensated for by varying levels of $\mathrm{O}_{2}$ extraction $(14,15,28,33)$. In studies showing a reduced muscle $\dot{\mathrm{V}}_{2}$, improved economy of force development has been proposed on the basis of the sparing of muscle phosphocreatine and unchanged lactate $(2,28)$, but this contrasts with the concept that NO increases oxidative phosphorylation efficiency $(10,23)$. In theory, our results would not support an improvement in efficiency of the phosphorylation system with combined inhibition of NOS and PG synthesis, since the suppression of mitochondrial respiration by Indo was most

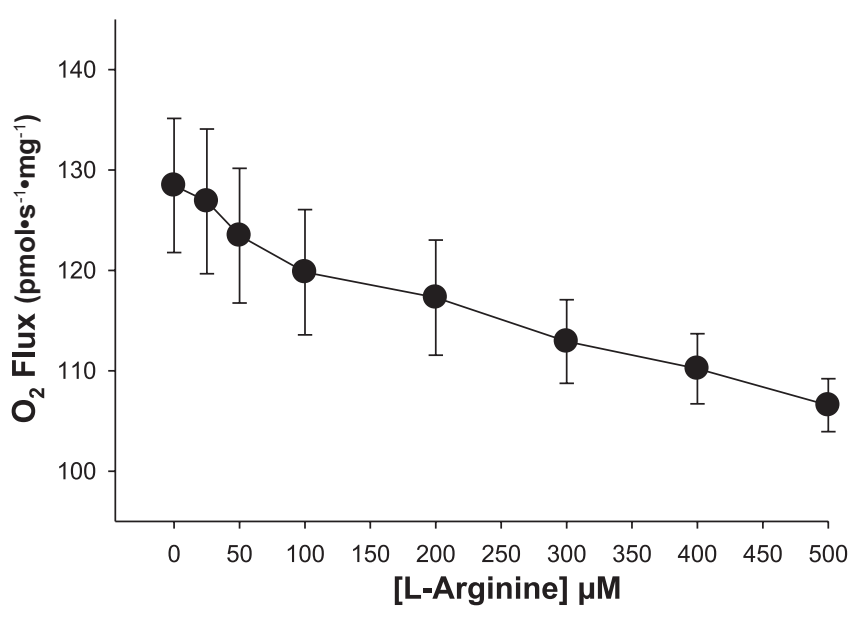

Fig. 4. Dose-response effect of L-arginine on state 3 mitochondrial respiration with electron supply to complex I + II in CON in the presence of ADP and neuronal NOS (nNOS) in rat skeletal muscle. 


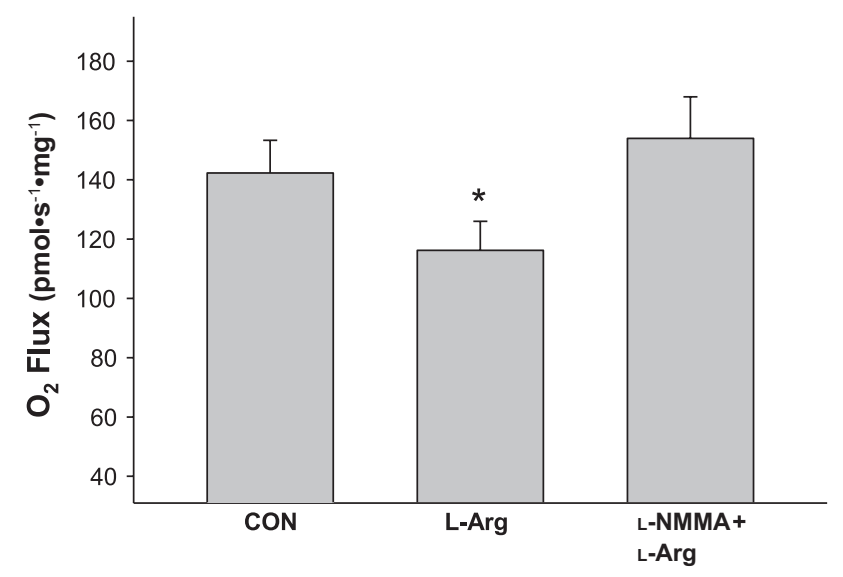

Fig. 5. The effect of NO (L-arginine + nNOS) and NOS inhibition (L-NMMA + L-arginine + nNOS) on mitochondrial state 3 (GS3) respiration with supply of glutamate and succinate in the presence of ADP in rat skeletal muscle. *Significant difference, $P<0.05$ from control.

pronounced at complex I, which has a higher $\mathrm{P}: \mathrm{O}$ ratio (3 protons pumped) compared with complex II (2 protons pumped). Accordingly, the higher substrate control ratio (GMS3/GM3) with indomethacin (Fig. 2A) indicates a proportionately greater reliance on complex II-supported respiration, which would elicit a lower efficiency or increase oxygen consumption for a given ATP synthesis. This upstream respiratory chain regulation together with the downstream effect of alleviating the inhibition of cytochrome- $c$ oxidase by NO would provide the explanation for a "higher" muscle $\dot{\mathrm{V}}_{2}$ for a given ATP production during combined inhibition of NOS and PG synthesis during exercise (14). However, our findings of a $15 \%$ reduction in state 3 respiration with combined inhibition are more in line with reports of a lower muscle $\dot{\mathrm{V}}_{2}$ during exercise $(15,28)$. The state 3 respiration rates of 5.4 and 4.7 $\mathrm{mmol} \cdot \mathrm{kg}^{-1} \cdot \mathrm{min}^{-1}$ for $\mathrm{CON}$ and Double Block in this study correspond identically by extrapolation to the values of 300 and $260 \mathrm{ml} / \mathrm{min}$ measured across the quadriceps in vivo under the same conditions (28). At a work rate of $30 \mathrm{~W}$, the external work rate to ATP synthesis rate is $\sim 28 \mathrm{~kJ} / \mathrm{mol}$. At a P:O ratio of 2.5 (29), the mitochondrial respiratory rate of 5.4 $\mathrm{mmol} \cdot \mathrm{kg}^{-1} \cdot \mathrm{min}^{-1}$ in CON matches the ATP demand for external work. Under the combined inhibition condition, a combined increase in efficiency of $\sim 13 \%$ from individual or combined components of phosphorylation ( $\mathrm{P}: \mathrm{O}$ ratio), the actomyosin ATPase reaction or mechanical efficiency would be required. A change in muscle efficiency has been suggested previously $(1,3,10,30)$, and it has been shown that changes in muscle ATP and ADP slow the actomyosin duty cycle and excitation (42).

An explanation for the finding of a higher muscle $\dot{\mathrm{V}}_{2}$ with combined inhibition of NOS and PG synthesis measured in vivo by PET (14) compared with a lower $\dot{\mathrm{V}}_{2}$ found across the whole limb by the Fick method $(15,28)$ could be due to differences in workload, measurement method, the concentration of inhibitor in tissue, and resulting mitochondrial proton leak in vivo. The simplest explanation is that the concentration of indomethacin that reached the muscle capillary level in the former PET study (14) was lower, allowing the effect of NOS inhibition to dominate. They used a dose of indomethacin less than half (50 vs. $120 \mu \mathrm{g} \cdot \mathrm{min}^{-1} \cdot \mathrm{kg}^{-1} \mathrm{leg}$ mass) that used in the present study. From a methodological standpoint, PET-derived blood flow with $\left[{ }^{15} \mathrm{O}_{2}\right] \mathrm{H}_{2} \mathrm{O}$ (radiowater) and oxygen extraction by inhaled ${ }^{15} \mathrm{O}_{2}$ measure responses in vessels where water molecules are exchanged, excluding resting muscle and other tissues. The mass of adipose, bone, connective tissue, and inactive muscle could represent a significant source of sampling disparity between PET and Fick measures across the limb, especially given the lower work rate in the former study, but this factor would have less comparative impact on the disparity of findings in this study since our measurements were on 2-5 mg of muscle.

In the ex vivo muscle respirometry preparation in this study, the mitochondria of blood vessels are included in the sampled muscle, and this tissue is not insignificant. The vasculature represents 3-4\% of body mass with an arteriolar mass of $\sim 0.4 \%$ (39). The $\dot{\mathrm{V}}_{2}$ of arterioles is relatively high $(\sim 1.5 \mathrm{ml}$ $\left.\mathrm{O}_{2} \cdot \mathrm{g}^{-1} \cdot \mathrm{min}^{-1}\right)$, and it has been shown that constriction and dilation alters $\dot{\mathrm{VO}}_{2}$ of the vasculature in tissue-perfused preparations. An increase in arteriolar $\dot{\mathrm{V}}_{2}$ from 0.9 to 1.8 $\mathrm{ml} \cdot \mathrm{g}^{-1} \cdot \mathrm{min}^{-1}$ has been reported in arterioles transitioning from relaxed to contracted states with vasopressin and norepinephrine (44). The opposite response (a lower $\mathrm{O}_{2}$ extraction) is observed in the same preparation with attenuated vasodilation by indomethacin and L-NMMA (11). The increase in $\dot{\mathrm{V}}_{2}$ of arterioles seen with purely vasoactive constriction seems clear (44). In contrast, the effect on arterioles of inhibiting NOS and PG synthesis (11) would elicit similar opposing effects as in muscle mitochondria, for example, less $\mathrm{O}_{2}$ demand by arterioles due to diminished vasoconstriction, lower $\mathrm{O}_{2}$ used in the formation of PG ( 2 molecules consumed in the cyclooxygenase reaction to produce hyrdoperoxy endoperoxide from arachidonic acid), and inhibition of arteriolar mitochondrial respiration by indomethacin concurrent with less inhibition of cytochrome- $c$ oxidase by NOS blockade with L-NMMA. The inhibition effects on these vessels are clearly within the detectable (picomolar) range with respirometry but may be excluded from the PET measures.

In summary, the results of this study indicate that indomethacin depresses state 3 mitochondrial respiration, especially at complex I of the electron transport chain. The finding that both state 3 respiration and uncoupled $\mathrm{O}_{2}$ flux is enhanced by NOS inhibition conforms to the view that NO exerts an inhibitory effect on the electron transport chain. The combined effects of NOS and PG synthesis inhibition are consistent with findings of a lower muscle $\dot{\mathrm{V}}_{2}$ during exercise in humans. The observation of an increase in muscle $\dot{\mathrm{V}}_{2}$ as found previously (14) in these conditions would be dependent on NOS inhibition exerting a more dominant local effect than indomethacin. The present findings indicate that blood flow and oxygen consumption during exercise can be regulated both at the level of the vasculature and in the adjacent muscle mitochondria by similar, parallel signals.

\section{Perspectives and Significance}

The findings in this study have both physiological and pharmacological implications for our understanding of the integrated control of $\mathrm{O}_{2}$ delivery and $\mathrm{O}_{2}$ consumption during exercise. This study raises the interesting question of what variable is regulated in the tight coupling between blood flow and metabolic demand. On the one hand, the PG + NOS 
inhibition pathways have a solid physiological basis in the control of vascular function setting the level of muscle vasodilation. On the other hand, if the locus of control by these same regulators is initiated in mitochondria, downstream from blood vessels, then the metabolic signal established at the level of the mitochondria could contribute to the regulation of the vasodilatory signal to fine-tune the match between blood flow and metabolic demand. This would represent a highly efficient control system governing metabolic work for the organism with the engagement of multiple organ systems in $\mathrm{O}_{2}$ delivery.

\section{ACKNOWLEDGMENTS}

We would like the subjects who volunteered for the study. The study was supported by Novo Nordisk Foundation.

\section{DISCLOSURES}

No conflicts of interest, financial or otherwise, are declared by the authors.

\section{AUTHOR CONTRIBUTIONS}

Author contributions: R.C.B., Y.H., and B.S. conception and design of research; R.C.B., T.F., Y.H., and B.S. performed experiments; R.C.B. and T.F. analyzed data; R.C.B., Y.H., and B.S. interpreted results of experiments; R.C.B. and T.F. prepared figures; R.C.B. drafted manuscript; R.C.B., Y.H., and B.S. edited and revised manuscript; R.C.B., T.F., Y.H., and B.S. approved final version of manuscript.

\section{REFERENCES}

1. Ameredes BT, Provenzano MA. Influence of nitric oxide on vascular resistance and muscle mechanics during tetanic contractions in situ. J Appl Physiol 87: 142-151, 1999.

2. Baker DJ, Krause DJ, Howlett RA, Hepple RT. Nitric oxide synthase inhibition reduces $\mathrm{O}_{2}$ cost of force development and spares high-energy phosphates following contractions in pump-perfused rat hindlimb muscles. Exp Physiol 91: 581-589, 2006.

3. Bangsbo J, Krustrup P, Gonzalez-Alonzo J, Saltin B. ATP production and efficiency of human skeletal muscle during intense exercise: effect of previous exercise. Am J Physiol Endocrinol Metab 280: E956-E964, 2001.

4. Barclay JK, Woodley NE. Nitric oxide synthase inhibitors do not alter functional hyperemia in canine skeletal muscle. Can J Physiol Pharmacol 72: 1035-1041, 1994.

5. Boushel R, Langberg H, Gemmer C, Olesen J, Crameri R, Scheede C, Sander M, Kjaer M. Combined inhibition of nitric oxide and prostaglandins reduces human skeletal muscle blood flow during exercise. $J$ Physiol 543: 691-698, 2002.

6. Brown GC, Borutaite V. Nitric oxide, cytochrome c and mitochondria. Biochem Soc Symp 66: 17-25, 1999.

7. Brown GC, Cooper CE. Nanomolar concentrations of nitric oxide reversibly inhibit synaptosomal respiration by competing with oxygen at cytochrome oxidase. FEBS Lett 356: 295-298, 1994.

8. Brunori M, Giuffre A, Sarti P, Stubauer G, Wilson MT. Nitric oxide and cellular respiration. Cell Mol Life Sci 56: 549-557, 1999.

9. Cleeter MW, Cooper JM, Darley-Usmar VM, Moncada S, Schapira AH. Reversible inhibition of cytochrome $c$ oxidase, the terminal enzyme of the mitochondrial respiratory chain, by nitric oxide. Implications for neurodegenerative diseases. FEBS Lett 345: 50-54, 1994.

10. Clerc P, Rigoulet M, Leverve \& Fontaine EX. Nitric oxide increases oxidative phosphorylation efficiency. J Bioenerg Biomembr 39: 158-166, 2007.

11. Curtis SE, Vallet B, Winn MJ, Caufield JB, King CE, Chapler CK, Cain SM. Role of vascular endothelium in $\mathrm{O}_{2}$ extraction during progressive ischemia in canine skeletal muscle. J Appl Physiol 79: 1351-1360, 1995.

12. Demaurex N, Scorrano L. Reactive oxygen species are NOXious for neurons. Nat Neurosci 12: 819-820, 2009.

13. Fariss MW, Chan CB, Patel M, Van Houten B, Orrenius S. Role of mitochondria in toxic oxidative stress. Mol Interv 5: 94-111, 2005.

14. Heinonen I, Saltin B, Kemppainen J, Oikonen V, Nuutila P, Knuuti J, Kalliokoski K, Hellsten Y. Skeletal muscle blood flow and oxygen uptake at rest and during exercise in humans: a PET study with nitric oxide and cyclooxygenase inhibition. Am J Physiol Heart Circ Physiol 300: H1510H1517, 2011.
15. Hillig T, Krustrup P, Fleming I, Osada T, Saltin B, Hellsten Y. Cytochrome P450 2C9 plays an important role in the regulation of exercise-induced skeletal muscle blood flow and oxygen uptake in humans. J Physiol 546: 307-314, 2003.

16. Jacob M, Bjarnason I, Rafi S, Wrigglesworth J, Simpson RJ. A study of the effects of indomethacin on liver mitochondria from rats, mice and humans. Aliment Pharmacol Ther 15: 1837-1842, 2001.

17. Jones AM, Wilkerson DP, Koppo K, Wilmshurst S, Campbell IT. Inhibition of nitric oxide synthase by L-NAME speeds phase II pulmonary. $\dot{\mathrm{V}}_{2}$ kinetics in the transition to moderate-intensity exercise in man. $J$ Physiol 552: 265-272, 2003.

18. Kindig CA, McDonough P, Erickson HH, Poole DC. Effect of L-NAME on oxygen uptake kinetics during heavy-intensity exercise in the horse. $J$ Appl Physiol 91: 891-896, 2001.

19. Kindig CA, McDonough P, Erickson HH, Poole DC. Nitric oxide synthase inhibition speeds oxygen uptake kinetics in horses during moderate domain running. Respir Physiol Neurobiol 132: 169-178, 2002.

20. King-VanVlack CE, Mewburn JD, Chapler CK, MacDonald PH. Endothelial modulation of skeletal muscle blood flow and $\mathrm{V}_{2}$ during lowand high-intensity contractions. J Appl Physiol 92: 461-468, 2002.

21. Krause MM, Brand MD, Krauss S, Meisel C, Vergin H, Burmester GR, Buttgerait F. Nonsteroidal anti-inflammatory drugs and a selective cyclooxygenase 2 inhibitor uncouple mitochondria in intact cells. Arthritis Rheum 48: 1438-1443, 2003.

22. Krause DJ, Hagen JL, Kindig CA, Hepple RT. Nitric oxide synthase inhibition reduces the $\mathrm{O}_{2}$ cost of force development in rat hindlimb muscles pump perfused at matched convective $\mathrm{O}_{2}$ delivery. Exp Physiol 90: 889-900, 2005.

23. Larsen FJ, Schiffer TA, Borniquel S, Sahlin K, Ekblom B, Lundberg JO, Weitzberg E. Dietary inorganic nitrate improves mitochondrial efficiency in humans. Cell Metab 13: 149-159, 2011.

24. Lodi R, Kemp GJ, Montagna P, Pierangeli G, Cortelli P, Iotti S, Radda GK, Barbiroli B. Quantitative analysis of skeletal muscle bioenergetics and proton efflux in migraine and cluster headache. J Neurol Sci 146: 73-80, 1997.

25. McLaughlin SG, Dilger JP. Transport of protons across membranes by weak acids. Physiol Rev 60: 825-863, 1980.

26. Mingatto FE, Santos AC, Uyemura SA, Jordani MC, Curti C. In vitro interaction of nonsteroidal anti-inflammatory drugs on oxidative phosporylation of rat kidney mitochondria: respiration and ATP synthesis. Arch Biochem Biophys 334: 303-308, 1996.

27. Moreno-Sanchez R, Bravo C, Vasquez C, Ayala G, Silveira LH, Martinez-Lavin M. Inhibition and uncoupling of oxidative phosphorylation by non-steroidal anti-inflammatory drugs: study in mitochondria, submitochondrial particles, cells, and whole heart. Biochem Pharmacol 57: 743-752, 1999.

28. Mortensen SP, Gonzalez-Alonso J, Damsgaard R, Saltin B, Hellsten Y. Inhibition of nitric oxide and prostaglandins, but not endothelialderived hyperpolarizing factors, reduces blood flow and aerobic energy turnover in the exercising human leg. J Physiol 581: 853-861, 2007.

29. Rasmussen UF, Rasmussen H, Krustrup P, Quistorff B, Saltin B, Bangsbo J. Aerobic metabolism of human quadriceps muscle: in vivo data parallel measurements on isolated mitochondria. Am J Physiol Endocrinol Metab 280: E301-E307, 2001.

30. Reid MB, Kobzik L, Bredt DS, Stamler JS. Nitric oxide modulates excitation-contraction coupling in the diaphragm. Comp Biochem Physiol A Mol Integr Physiol 119: 211-218, 1998.

31. Sahach VF, Bohuslavs'kyi A, Dmytriieva AV, Nadtochii SM. Role of nitric oxide and mitochondrial permeability pore in changes of oxygen consumption in the working skeletal muscle. Fiziol Zh 50: 19-26, 2004.

32. Schrage WG, Joyner MJ, Dinenno FA. Local inhibition of nitric oxide and prostaglandins independently reduces forearm exercise hyperaemia in humans. J Physiol 557: 599-611, 2004.

33. Schrage WG, Wilkins BW, Johnson CP, Eisenach JH, Limberg JK, Dietz NM, Curry TB, Joyner MJ. Roles of nitric oxide synthase and cyclooxygenase in leg vasodilation and oxygen consumption during prolonged low-intensity exercise in untrained humans. J Appl Physiol 109: 768-77, 2010.

34. Schweizer M, Richter C. Nitric oxide potently and reversibly deenergizes mitochondria at low oxygen tension. Biochem Biophys Res Commun 204: $169-175,1994$.

35. Shen W, Xu X, Ochoa M, Zhao G, Bernstein RD, Forfia P, Hintze TH. Endogenous nitric oxide in the control of skeletal muscle oxygen extraction during exercise. Acta Physiol Scand 168: 675-686, 2000. 
36. Somasundaram S, Rafi S, Hayllar J, Sigthorsson G, Jacob M, Price AB, Macpherson A, Mahmod T, Scott D, Wrigglesworth JM, Bjarnason I. Mitochondrial damage: a possible mechanism of the "topical" phase of NSAID-induced injury to the rat intestine. Gut 41: 344-353, 1997.

37. Stumpe T, Decking UK, Schrader J. Nitric oxide reduces energy supply by direct action on the respiratory chain in isolated cardiomyocytes. Am J Physiol Heart Circ Physiol 280: H2350-H2356, 2001.

38. Tsai AG, Frieseneicker B, Mazzoni MC, Kerger H, Buerck DG, Johnson PC, Ingtaglietta M. Microvascular and tissue $\mathrm{O}_{2}$ gradients in rat mesentary. Proc Natl Acad Sci USA 95: 6590-6595, 1998.

39. Tsai AG, Johnson PC, Intaglietta M. Oxygen gradients in the mircrocirculation. Physiol Rev 83: 993-963, 2003.

40. Turrens JF. Mitochondrial formation of reactive oxygen species. $J$ Physiol 552: 335-344, 2003.
41. Ward ME, Hussain SN. Effect of inhibition of nitric oxide release on the diaphragmatic oxygen delivery-consumption relationship. J Crit Care 9: 90-99, 1994

42. West TG, Curtin NA, Ferenczi MA, He ZH, Sun YB, Irving M, Woledge RC. Actomyosin energy turnover declines while force remains constant during isometric muscle contraction. J Physiol 555: 27-43, 2004.

43. Xie YW, Wolin MS. Role of nitric oxide and its interaction with superoxide in the suppression of cardiac muscle mitochondrial respiration. Involvement in response to hypoxia/reoxygenation. Circulation 94: 2580 2586, 1996.

44. Ye JM, Colquhoun EQ, Clark MG. A comparsion of vasopressin and noradrenaline on oxygen uptake by perfused rat hindlimb, intestine and mesenteric arcade suggests that it is in part due to contractile work by blood vessels. Gen Pharmacol 21: 805-810, 1990.

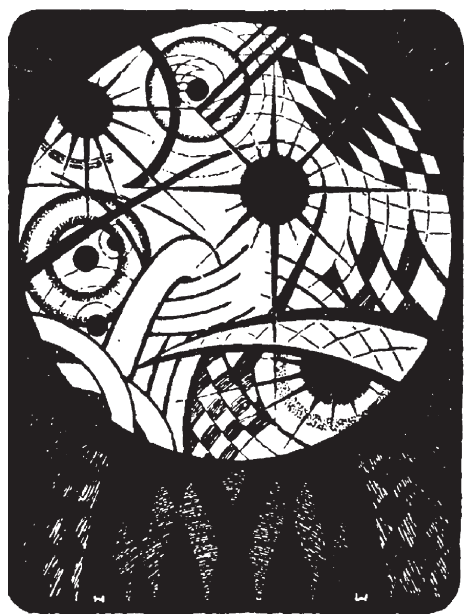

